\title{
Loss of Drosophila Hyperplastic disc promotes massive cell death and germline atrophy in oogenesis
}

\author{
Iuliia Aleksandrovna Galimova \\ IMCB SB RAS, Novosibirsk, Russia \\ galimova@mcb.nsc.ru
}

\author{
Natalia Vladimirovna Dorogova \\ ICG SB RAS, Novosibirsk, Russia \\ dorogova@bionet.nsc.ru
}

Svetlana Aleksandrovna Fedorova

ICG SB RAS, Novosibirsk, Russia

fsveta@bionet.nsc.ru

\author{
Elena Ustinovna Bolobolova \\ ICG SB RAS, Novosibirsk, Russia \\ elbol@bionet.nsc.ru
}

\begin{abstract}
Drosophila hyd is ortholog of the mammalian tumor suppressor $E D D$ that implicated in a wide variety of cellular processes and its regulation is impaired in various types of tumors. It is a member of highly conservative HECT family of E3 ubiquitin ligases that directly transfers the ubiquitin to targeted substrates. Hyd is required for the regulation of cell proliferation during development, mutations in the hyd gene resulting in developmental abnormalities including adult sterility. Reduction of hyd expression results in large-scale germ cells death at different stages of oogenesis. Germ cells lacking Hyd demonstrated a slowdown in the growth and development during mid-oogenesis, leading to the total cell death due to metabolic impairment.
\end{abstract}

Keywords - hyperplastic disc gene, oogenesis, cell death, E3 ubiquitin ligase, germ cells, drosophila

\section{Motivation and Aim}

One of the important aspects of carcinogenesis investigation deals with tumor suppressor genes that control the cellular constancy of the body and prevent the formation of tumors. Drosophila melanogaster hyperplastic disc gene $(h y d)$ is ortholog of the mammalian tumor suppressor EDD (E3 identified by differential display, EDD/UBR5/hHyd, hereafter called EDD). EDD implicated in a wide variety of cellular processes and its regulation is impaired in various types of tumors. Hyd, as well as EDD, has E3 ubiquitin ligase activity and is critical for cell proliferation and differentiation $[1,2]$. Mutations in hyd gene display a range of developmental phenotypes, including imaginal disc abnormalities [3], larval lethality, sterility [4], premature photoreceptor differentiation [5], failed oogenesis [6], and defective spermatogenesis [7]. The clone analysis identified that Hyd negatively regulates expression of Hedgehog (Hh) and Decapentaplegic (Dpp) in eye and wing imaginal discs [5]. Here, we study the Hyd function in Drosophila oogenesis. We showed that the reduction of hyd expression by mutations or RNA interference results in large-scale germ cells death at different stages of oogenesis. This cell death does not depend on Dpp or Hh signaling.

\section{Methods}

We used Bloomington Drosophila stocks: kni hyd15 e1 /TM3, Sb1 (3718) and yw; PBac \{3HPy+\} hydC017/TM3, Sb1 Ser1 (16256) as the source of mutant hyd alleles. To suppress hyd expression in different ovarian cells we used the hyd RNA interference. To induce the RNA interference we combined UAS-hyd-RNAi transgene with a set of different ovarianspecific GAL4 drivers. We performed a detailed cytological analysis of oogenesis using fluorescent and electronic microscopy.

\section{Results and Discussion}

For the cytological analysis of the mutant effect in oogenesis, viable females carrying the interallelic combination hydC017/hyd15 were used. hydC017/hyd15 females are sterile and lay only single eggs from which embryos do not develop. The gonads of such females mainly contain partially or completely reduced ovaries: either with a small number of egg chambers, or without them at all

Antibodies staining of germline cells marker, Vasa protein, showed that more than $90 \%$ of the ovaries consisted of ovarioles, either containing no germline cells or with single egg chambers. Although gonads form normally in embryos and larvae, but their internal structure degrades at later stages, only part of the cells gives rise to cysts that form a normal egg chamber. However formed egg chambers also degrade during oogenesis, only $1 \%$ of them develop into an egg. Thus, hyd mutations affect the viability of cells involved in oogenesis and egg chamber forming. Stem cells also die, and, as a result, ovarioles without germ cells are formed. Also cells die in germarium and egg chambers at different stages of development.

Oogenesis defects detected in hydC017/hyd15 are associated with a decrease in hyd gene expression. Quantitative analysis of mRNA using real-time PCR showed that the level of gene expression is statistically different and about 2 times lower in the mutant than in the control (wild type).

The association of cell death with a decrease of the hyd activity is confirmed by our data obtained using hyd RNA interference (hyd-RNAi). For this experiment, we used the $U A S / G A L 4$ system. In order to ectopically suppress the hyd gene expression in different ovary cells, tissue-specific drivers were selected: nanos-GAL4, oskar-GAL4 - for germline cells, 7023-GAL4, 7024-GAL4, 36287-GAL4 - for somatic cells. Only combination $U A S$-hyd-RNAi with germline specific drivers, nanos-GAL4 and oskar-GAL4, resulted in massive cell death and ovarian atrophy, which corresponded to the phenotypic manifestation of mutations.

As the nanos-GAL4 starts to express in primordial germline cells from embryogenesis, in the nanos-GAL4/UAShyd-RNAi females, germ cell death occurred before reaching the adult stage. Electron microscopy revealed signs of cell death everywhere in the germ line of such female at the end of 3rd larval stage. Normally, at this stage, germ cells actively proliferate and increase in their number without subsequent differentiation. Cell death at this stage may be associated with misregulation of factors that control the cell cycle, cell death pathways, nuclear envelope and cytoskeleton remodulating. 
The oskar-GAL4 is activated at stages 3-4 of oogenesis, and in oskarGAL4/UAS-hyd-RNAi females, germ cell death occurred in mid-oogenesis. In this case, after stage 4-5, germ cells slowed down in the development. Although they slightly increased in size, the oocyte does not grow normally and does not increase in volume relative to the nurse cells area, compared wild type. The developmental arrest ended with the total $(100 \%)$ death of the egg chambers. Electron microscopic analysis showed that the Hyd lack did not lead to the anomalies in the cell ultrastructure. Germ cells degradation occurred at the same stage of oogenesis as in the wild type and did not differ morphologically. Massive germ cells death and egg chambers in mid oogenesis were also observed when the expression of inhibitors of apoptosis Bruce and Diap was reduced [8]. However, in the Hyd loss background, the germ cells death occurred after a slowdown in the development and growth, which indicates a gradual extinction of cellular metabolism and a decrease in viability. We believe there is an effect similar to a protein deficiency or environmental stress. It is known that starvation and lack of proteins activate an oogenesis checkpoint triggering the mechanism of cell death [9]. Probably, the lack of E3 ubiquitin ligase Hyd leads to a disruption in the ubiquitination mechanism of the protein substrates responsible for the metabolism and synthetic activity of germ cells, causing cell death due to deficit of energy resources.

\section{ACKNOWLEDGMENT}

Supported by the RFBR (20-04-00496), and by ICG SB RAS budget Project (0324-2019-0042-C-01).

\section{REFERENCES}

[1] Clancy J.L. et al. (2003) EDD, the human orthologue of the hyperplastic discs tumour suppressor gene, is amplified and overexpressed in cancer. Oncogene. 22: 5070-5081.

[2] Shearer R.F.et al. (2015) Functional Roles of the E3 Ubiquitin Ligase UBR5 in Cancer. Mol Cancer Res. 13(12):1523-1532.

[3] Martin P. et al. (1977) Studies of 1(3)c43hs1 a polyphasic, temperaturesensitive mutant of Drosophila melanogaster with a variety of imaginal disc defects. Dev Biol. 55:213-232.

[4] Mansfield E. et al. (1994) Genetic and molecular analysis of hyperplastic discs, a gene whose product is required for regulation of cell proliferation in Drosophila melanogaster imaginal discs and germ cells. Dev Biol. 165:507-526.

[5] Lee J.D. et al. (2002) The ubiquitin ligase Hyperplastic discs negatively regulates hedgehog and decapentaplegic expression by independent mechanisms. Development. 129:5697-5706.

[6] Szabad J. et al. (1991) Requirement for cell-proliferation control genes in Drosophila oogenesis. Genetics.127:525-533.

[7] Pertceva J.A. et al. (2010) The role of Drosophila hyperplastic discs gene in spermatogenesis. Cell Biol. Int.34:991-996.

[8] Nezis I.P. et al. (2010) Autophagic degradation of dBruce controls DNA fragmentation in nurse cells during late Drosophila melanogaster oogenesis. .J Cell Biol. 190(4):523-531.

[9] Jenkins V.K. et al. (2013) Diversity of cell death pathways: insight from the fly ovary.Trends Cell Biol. 23(11):567-574. 